Characteristics and outcome indicators in a specialist inpatient intellectual disability unit: an independent sector experience

Mike Apio*, Witold Skalbania, Muhammad Aamer Khan and Bolanle Lotsu

Eldertree Lodge, The Huntercombe, Ashley

${ }^{*}$ Corresponding author.

doi: 10.1192/bjo.2021.816

Aims. To elucidate critical elements for effective outcomes in patients with complex and challenging behaviours admitted to specialist inpatient 'locked rehabilitation' intellectual disability unit (LRU).

Background. People with intellectual disability of varying severity with or without associated mental disorder are at risk of deterioration presenting with problem behaviours at critical times of transition. In the context of their pre-set neurocognitive deficits, protective factors during early development include a robust psychosocial 'parenting' environment that optimises their strengths through nurturing and embedding a positive mind-set. Such environment is critical for the development of resilience as against reliance on external factors with high likelihood of change. The effect of early exposure to prenatal and or postnatal childhood adversities is a common denominator. The experience of abuse; from deprivation and neglect to physical violence and indeed sexual trauma predisposes to further perturbation and kindling effect on risks for early and later onset affective disorders. Specialist ID services become critical to the resetting of a distorted premorbid neuronal circuitry. A biopsychosocial approach to recreating a stable base and environmental enrichment may offer opportunities for enhancing neurocognitive remediation and enhance prosocial skills. Indicators for better outcomes may offer scope for focused intervention. This review highlights extent patients progress (response to treatment and symptom remission), length of Stay and discharge pathway could be predicated on their engagement with offered structured therapeutic activities.

Method. Using a mixed model approach, 12-months data regarding patient characteristics, elements from HoNOs LD, with patient's self-reported experience and utilization of therapy, progress of patients in the service were reviewed to elucidate factors that may predict improved outcomes..

Result. Of 48 patients, 18 females and 30 males identified in the 12-months from January 2019, 7 females were discharged/transferred with one stepped up to LSU and another side-moved to a LRU. 6 have identified places and 5 require ongoing care. Of the males, 8 were discharged and 5 have identified placements. 16 inpatients with support completed questionnaires (10 males, 6 females). Majority identified structured therapeutic activities as helpful in their progress. Data for length of stay ranged from 12 to over 120 months with a mean of 31 months ignoring potential discharges.

Conclusion. Findings suggest patients able to engage in structured therapeutic activities in conjunction with concordance to treatment are more likely to progress earlier in their care.

\section{A new service model in East Lothian community learning disability team: evaluation of service with and without specialist positive behaviour support team \\ Saima Asif ${ }^{1 \star}$ and Andrew McKechanie ${ }^{2}$ \\ ${ }^{1}$ ST5, NHS Lothian Learning Disability Service and ${ }^{2}$ Consultant Psychiatrist, NHS Lothian Learning Disability Service ${ }^{\star}$ Corresponding author.}

doi: 10.1192/bjo.2021.817
Aims. To evaluate the provision of services to patients with challenging behaviour in East Lothian Community Learning Disability population with and without specialist behaviour support team.

Background. Behaviour that proves to be a challenge to manage (Challenging behaviour) is not uncommon in adults with intellectual disability and has a reported prevalence of $10-15 \% .1,2$

Positive behaviour support (PBS) is recommended as evidencebased intervention for adults with intellectual disability who have challenging behaviour. East Lothian community learning disability team (CLDT) underwent a change in service model for people with challenging behaviour. This change followed a Health and Social care partnership agreement that behaviour support and management could be provided by multidisciplinary CLDT rather than region-wide specialist team.

Method. Data collection was split into two cycles. First cycle looked retrospectively at six months prior to exit of Specialist Positive Behaviour Support Team (SPBST). Second cycle looked prospectively at 6 months after exit of SPBST.

In first cycle, data were collected doing retrospective review of cases known and referred to SPBST. This included calculating time spent on each individual case by SPBST and by CLDT. SPBST provided information in the form of hours spent on each individual case for patients identified by them. For CLDT, electronic medical records system (TRAK) was used by looking at appointment entries on TRAK. For second cycle, newly developed Complex Behaviour pathway was used to identify the patients. Data were collected by using TRAK system as in the first cycle for CLDT.

Data collected in both cycles was compared at the end of second cycle.

Result. In first cycle, 5 patients were managed jointly by SPBST and CLDT in 96.4 hours over six months and average clinical time spent on each patient was 19 hours. SPBST spent a total of 59 hours and CLDT spent 40 clinical hours. In second cycle, 12 patients were managed by CLDT alone in 130 hours over six months and average clinical time spent on each patient was nearly 11 hours.

Conclusion. Results of this evaluation suggest that SPBST had been providing significant contribution to East Lothian CLDT not only with their expertise but also with clinical time. More than $50 \%$ of total clinical time spent on the patients with challenging behaviour in first cycle, was provided by SPBST. This is also evidenced in second cycle where there is an increase in clinical time of some professions when SPBST was withdrawn.

The use of benzodiazepines and Z-drugs in the Acute Psychiatric Unit at Cavan General Hospital

Salah Ateem ${ }^{1 \star}$ and Rachael Cullivan ${ }^{2}$

${ }^{1}$ St. Davnet's Hospital and ${ }^{2}$ Cavan General Hospital

${ }^{*}$ Corresponding author.

doi: 10.1192/bjo.2021.818

Aims. Benzodiazepines and Z-drugs are used frequently in acute psychiatric wards, however long-term administration can result in undesirable consequences. Guidelines recommend prescription of the lowest effective dose for the shortest period and if possible to prescribe "as required" rather than regularly. The 25-beded inpatient unit at Cavan General Hospital admits adult patients requiring acute care from the counties of Cavan and Monaghan. Admissions are accepted from four community mental health teams, two psychiatry of old age teams and the rehabilitation and mental health of intellectual disability teams. In order to evaluate 